\title{
LA ESTRUCTURA HOSPITALARIA, LOS CUIDADOS Y CUIDADORES EN LOS HOSPITALES EXTREMEÑOS EN LA BAJA EDAD MEDIA
}

\author{
Alvaro Barra, $\mathrm{M}^{\mathrm{a}} \mathrm{P}$; Morlans Loriente, $\mathrm{M}^{\mathrm{a}} \mathrm{J}$; De la Peña Tejeiro, E; Gómez Galán, \\ R; Garrido González, J.
}

Centro de Realización: Departamento de Enfermería. Universidad de Extremadura. Correspondencia: Esperanza de la Peña Tejeiro. Escuela Universitaria de Enfermería Carretera de Valverde Km. 2 - 06800 - Mérida (Badajoz)

Tel: 9243032 65; Fax: 924302666

\section{RESUMEN}

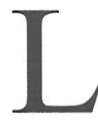
a filosofía Cristiana, las grandes batallas con fines religiosos, la baja economía y la heterogeneidad de culturas, van a ser las características mas relevantes que marcaron la estructura de los hospitales, los recursos de los mismos y los cuidados que en ellos se realizaban. En estas casas u hospitales, se atendían a los pobres, enfermos, peregrinos, indigentes y niños huérfanos. Los cuidados principales que se impartieron fueron la alimentación, reposo y administración de ungüentos y pócimas. Todas estas actividades se realizaban sobre la base religiosa de la caridad y amor al prójimo.

Los cuidadores eran, en su mayoría clérigos y en ocasiones personas laicas motivadas por los principios ya mencionados en el párrafo anterior. Los clérigos a parte de cuidar, desarrollaban todas las actividades relacionadas con la gestión y organización del centro, dejando para los laicos, todas aquellas labores de limpieza, y preparación de alimentos.

HOSPITAL STRUCTURE, CARE AND CARERS IN EXTREMADURA HOSPITALS DURING THE LOW MIDDLE AGES ALVARO BARRA, MP ET AL.

Dhe Christian philosophy, the battles with religious content, poor economy and cultural heterogeneity, were relevant characteristics that influenced hospitals structure, resources and care. Paupers, the sick, pilgrims, homeless and orphans were assisted in this homes or hospitals.
Food, rest and administration of ointments and medicines were the main ways of being looked after. All these activities were carried out under the religious principles of charity and love to others.

Carers were mainly clergymen or sometimes lay people motivated by the above mentioned principles. Clergymen, besides being carers, were in charge of tasks related to management and organisation of the place, leaving the cleaning and cooking for the laic.

\section{PALABRAS CLAVES.}

Hospital, cuidadores, cuidados.

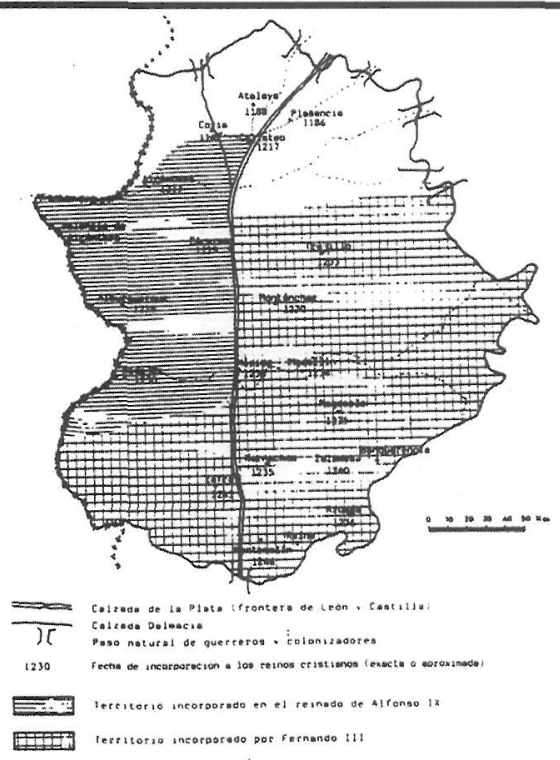

Figura 1: La expansión cristiana por el territorio extremeño. 


\section{INTRODUCCIÓN}

$\mathrm{E}$ n sus más remotos orígenes, dar cuidados, era una actividad ligada a cualquier modo de vida. De forma que puede considerarse inseparable a la lucha por la supervivencia de los seres humanos y a la continuidad de la vida en grupo y/o en comunidad. (1)

La Edad Media, época en la que nos vamos a centrar para la realización de nuestro estudio, va a estar marcada por las diversas invasiones, una economía autárquica, el empobrecimiento social, las grandes epidemias, el fanatismo religioso y el oscurecimiento artístico y cultural. Estos fenómenos, a grandes rasgos, son los que condicionaron la forma de vida y las conductas de la sociedad de la España del Medievo. Otra de las características que debemos distinguir, por su importancia histórica, es la consolidación de la Iglesia y la filosofía Cristiana. Estos hechos condujeron a la gran proliferación de ermitas, Iglesias, hospitales y casas de beneficencia para albergar entre otros, a los pobres, enfermos y débiles de espíritu. Todos ellos asistidos por un grupo social fuertemente marcado por las ideas religiosas y más concretamente por los principios de caridad y amor al prójimo que, sin duda, se manifestaban en su forma de vida, de relacionarse y de cuidar. Esta última actividad, resaltaría por la gran demanda social del momento.

Para realizar nuestro estudio, hemos recopilado toda la documentación que recogía la información respecto a los hospitales extremeños y más específicamente la que hace referencia a dos provincias: Cáceres, en torno a la villa de Montánchez y Badajoz, en la que nos centraremos en la ciudad de Mérida y pueblos circundantes.

El objetivo de nuestro trabajo, es revisar las características más importantes y comunes a los diez hospitales extremeños seleccionados, así como descubrir los cuidadores y los cuidados que se realizaban en los mismos.

La metodología utilizada ha consistido en el análisis e interpretación de los datos de las fuentes documentales que datan de los siglos XIII y XIV y que recogen información de veintiocho pueblos $\mathrm{y}$ veinticuatro hospitales.

\section{CARACTERÍSTICAS ESTRUCTURALES DE LOS HOSPITALES.}

A pesar de que en la información que hemos manejado, las descripciones son muy poco específicas, las características que vamos a describir se cumplían en los siguientes hospitales: Montánchez, Arroyomolinos, Montijo, Alange, Mérida, Lobón, San Pedro, Almoharin, Garrobilla Y Torremocha.

La distribución territorial de los hospitales se realizaba a merced de las iniciativas de caridad, así lo ponen de manifiesto los estudios realizados sobre la ruta Jacobea, los monasterios y los hospitales, quedando patente por el volumen numérico de estas instituciones con respecto al resto de las estructuras arquitectónicas. (2)

La fundación y donación van a depender de todas aquellas personas que su poder adquisitivo les permitía colaborar en la causa o por aquellos que su buena voluntad y solidaridad con los pobres les motivaba a realizar una buena obra social. así nos hemos encontrado que el hospital de Lobón fue fundado y donado por Diego de Alvarado en torno a 1470, año, en el que construyó la torre de Lobón como símbolo de su poder; el de Arroyomolino por Pedro de Sanabria y el de Alange por García López Pacheco, que en la primera y segunda data dicen: " ay en dicha villa un ospital pobre e desbaratado de casa e de ropa." "Don García López Pacheco, comendador que fue de la dicha villa, mandó que en una casa que él tenía en la calle principal que se dice Juan Gómez, fuese ospital... " . (3) El resto de los centros los instituyeron un buen hombre, una buena mujer o lo silencia la documentación.

En ocasiones la fundación del hospital requería nuevas construcciones, pero en la mayoría de los casos sé ergía en una vivienda donada para tal fin, ya que no existía ninguna normativa que especificara las características que debían reunir estos centros de acogida. En otros casos, el edificio se modelaba a las necesidades del mismo. Lógicamente esta situación obligaba a identificar la casa u hospital, para ello en la puerta principal ponían unas tablas pintadas o la imagen de la Virgen o del santo en cuya memoria se fundaba el hospital, así como un letrero que perpetuaba a sus fundadores y dejaba constancia de sus obras de caridad. 
La arquitectura del hospital medieval extremeño, no va a experimentar cambios a lo largo de su existencia, máxime al estar sostenido por escasos recursos económicos que van a condicionar el numero de habitaciones y en algunas ocasiones el esquema de agrupación de ellas. Esta circunstancia, en cada región lo solucionaban con procedimientos diferentes, en Extremadura, la solución típica en planta, era un pasillo central con habitaciones a ambos lados del mismo, con un extremo opuesto en el que se ubicaba el corral (4), que generalmente se encontraba en las traseras de los edificios, aunque hubo alguna excepción como en San Pedro, Montánchez y Alamohain que se situaron en la delantera. Sólo seis tuvieron caballeriza, aunque nos imaginamos que deberían de contar con pocos animales de tiro. Como dato pintoresco documentando, la casa de Arroyo tenía una "borrica".

Los alzados de mayor prestancia exterior correspondieron a los edificios de Montijo y Lobón, pues ambos tenían delante de la casa un portal de arcos de ladrillo para cumplir la grata misión de acoger a los menesterosos, tanto para que recibiesen el cálido sol invernal, como para que se resguardasen del calor veraniego. Esta característica también la desempeñaban los portales de San Pedro, Arroyomolinos y Zarza de Alange. El resto, tenía un alzado sencillo común al estilo de la época.

De las dimensiones de las habitaciones, no hemos encontrado ninguna referencia, ni tampoco la superficie ocupada por ellas. En la información manejada, se puede extaer la siguiente nota, que corresponde al hospital de la Garrobilla: "...es una casa que tiene una delantera luenga, e a un lado una cámara en la cual avia una cama armada e delante de la delantera ay otra pieca grande en la gual avia dos cámaras". (3) Dado que las descripciones sobre los hospitales son muy escuetas, como dijimos anteriormente, es posible que esta distribución se encontrase en algunas de ellas, aunque sólo enumeren las cámaras, ya que estos espacios eran comunes en las casas de alto rango, y los hospitales, en ocasiones, fueron edificios que se donaron y utilizaron para este fin. Otra característica común es que existía una habitación para el rector de la institución y una cama para el hospitalero.
Los materiales de construcción utilizados por antonomasia fueron el barro, la piedra, la madera, la cana y la jara. El ladrillo solo se empleo en los arcos de los porticos de Montijo, Lobón, y Alange.

Las techumbres, en la mayoría de ellos fueron de cana junta sobre madera de pino o sobre madera de castano, enke estos elementos y las tejas habia una torta de cal, excepto en el de Alange que se techo solo con madera de pino.

Para los pavimentos, se alternaba la cal y la piedra; los pasillos a veces estaban empedrados.

Las puertas tenían cerraduras y como dato curioso estaban protegidas con cadenas, se puede deducir que el objetivo de esto consistia en el control y aislamiento de los pobres y enfermos, ya que era uno de los fines que perseguian los centros asistenciales en la Baja Edad Media.

La iluminación estuvo sometida a los imperativos de la época medieval, entre los cuales el mas restrictivo era la limitación de la luz. La luz natural se debia a pequenas ventanas que generalmente estaban situadas en algunos de los lados que daban a la calle. El resto de la iluminación se cubria con apliques con velas o farolillos de gasolina u otra sustancia. (4)

Con respecto a la capilla, sólo la tuvieron el hospital de Nuestra Señora de Mérida y el de Nuestra Señora de la Piedad de Torremocha, al que corresponde la siguiente referencia: " dicha ermita esta un ospital..". (3)El hospital de Nuestra Señora de Mérida, pone de manifiesto en la traza del ala derecha de la casa, la existencia de una capilla que se comunicaba a través de una ventana grande con una reja de madera con la sala donde se encontraban los enfermos.

Para concluir este apartado mencionaremos que el hecho de que los hospitales fuesen pequeños, seguramente fuera la causa de que en situaciones de gran demanda de cuidados (epidemias), para acoger a todos los necesitados, procedían a colocar una cama encima de la otra, a modo de literas, hecho muy natural en la Edad Media. (5)

\section{CUIDADORES Y CUIDADOS}

Respecto al tema, que a continuación vamos a tratar, las fuentes documentales no son muy explícitas al respecto, ya que los estudios realizados por los medievalistas sobre distintas instituciones hos- 
pitalarias se centran principalmente en aspectos generales relacionados con sus posesiones y su actividad económica.

En los archivos históricos de la biblioteca municipal de Mérida, (3) se han encontrado algunos legajos, que hacen referencia a la fundación de estos hospitales y dejan constancia del número de personas que se encargaban de cuidar a los enfermos, utilizando diferentes nombres como hospitaleros, ministrates, cuidadores y en algunos casos y más concretamente a finales de la Edad Media los llamaban enfermeros.

Los términos de cuidadores y enfermeros, se utilizaban tanto en masculinos como en femenino, ésto hace pensar que por el periodo que estamos viendo y más concretamente sobre la base del cristianismo, en la mayoría de estos centros los hombres cuidaban a los enfermos varones y las mujeres a las enfermas.

Si nos centramos en los principios que motivaban el cuidado de los enfermos, que no eran otros como el amor al prójimo y la caridad, podemos deducir que en su mayoría, los cuidadores fueron personas del clero, completando la plantilla personas laicas entregadas a los principios religiosos de la filosofía cristiana. Los laicos, entre otras actividades (limpieza, cocina etc.), se dedicaban a la actividad de cuidar y los clérigos dividian su tiempo en dispensar cuidados y realizar actividades de organización y gestión de los hospitales.

Quizás por rebeldía a todo lo que representaba la forma de vida de la época romana, la higiene, no era una de las necesidades básicas más cuidadas en la Edad Media, se centraban principalmente en la alimentación y en la administración de pócimas, ungüentos y brebajes. Sin olvidar, el tratamiento del espíritu y el consuelo, a los enfermos moribundos y terminales, siempre impartidos desde la mentalidad religiosa, que sin lugar a duda con la evolución a lo largo de la historia de Enfermería, es lo que hoy realizamos desde la perspectiva humanista implícita en la profesión de Enfermería.

Los cuidados relacionados con la alimentación era la base de la atención al enfermo hospitalizado, adquiriendo las personas cuidadoras la responsabilidad de que la dieta fuera la indicada por el médico y que se administrase al enfermo con la preparación adecuada. Los alimentos que más se utiliza- ban eran los más producidos en Extremadura, entre los que se encuentran las legumbres, verduras, aceites y carnes, no teniendo constancia de la leche y sus derivados.

Muy relacionados con la nutrición del enfermo, eran los productos de botica destinados para la preparación de pócimas y brebajes, que podían ser de uso tópico o ingeridos por el propio enfermo en las comidas. Entre estos productos se hallan las frutas, los dulces, confituras, especies, almendras, pasas, regaliz, miel, anís, pimienta y sobre todo el aceite de oliva.

Siempre que les fuera posible, otra de las preocupaciones, era cambiar la ropa de la cama o ventilar el jergón donde acogían el cuerpo del enfermo, como lo pone de manifiesto la nota que hace referencia a la casa de Montánchez y que dice: "tiene fasta tres camas de ropa.. ${ }^{66}$. (3) expresión denota que no era muy corriente que hubiera provisión de camas para todos los necesitados.

\section{CONCLUSIONES}

Las circunstancias políticas, económicas y principalmente religiosas, marcaron la necesidad de crear hospitales que en su mayoría eran donados por ciudadanos privilegiados, que su condición económica y social les permitía ceder algunas de sus casas o palacios para el asilo de peregrinos, personas abandonadas y cuidado de los enfermos.

Sin lugar a duda, una vez más queda reflejado en la historia, el papel tan relevante que los cuidadores representaron en el sistema social, que sin lugar a duda en este periodo adquirió más protagonismo por la gran demanda que género las epidemias, el hambre y la miseria que sufrió la población.

La filosofía Cristiana, a través de sus seguidores, marcó e introdujo en los cuidados el aspecto espiritual y el consuelo moral a los enfermos, condenados a morir y débiles de espíritu.

La época de la construcción de los edificios y el tipo de material utilizado sugieren el estilo almudéjar de dichas construcciones, adaptadas luego a la utilidad y objetivos de los hospitales.

Los cuidados básicos se basaron principalmente en dar cobijo, un lecho, ropa limpia y alimentación a todos aquellos que lo necesitaran. 


\section{BIBLIOGRAFÍA}

1- SANTO TOMÁs, M (1994). enfermería Fundamental. Master de Enfermería. Barcelona Ediciones Mosson S. A. (Capítulo a Libro).

2- Martín, Jl y García, MD (1985). Historia de Extremadura. Badajoz. Editotial Universitas. (Capítulo a Libro).

3- Ruiz Mateos, A. (1990) "Hospitales Extremeños en la Baja Edad Media" Editorial. Estudios Extremeños. (Artículo de revista).
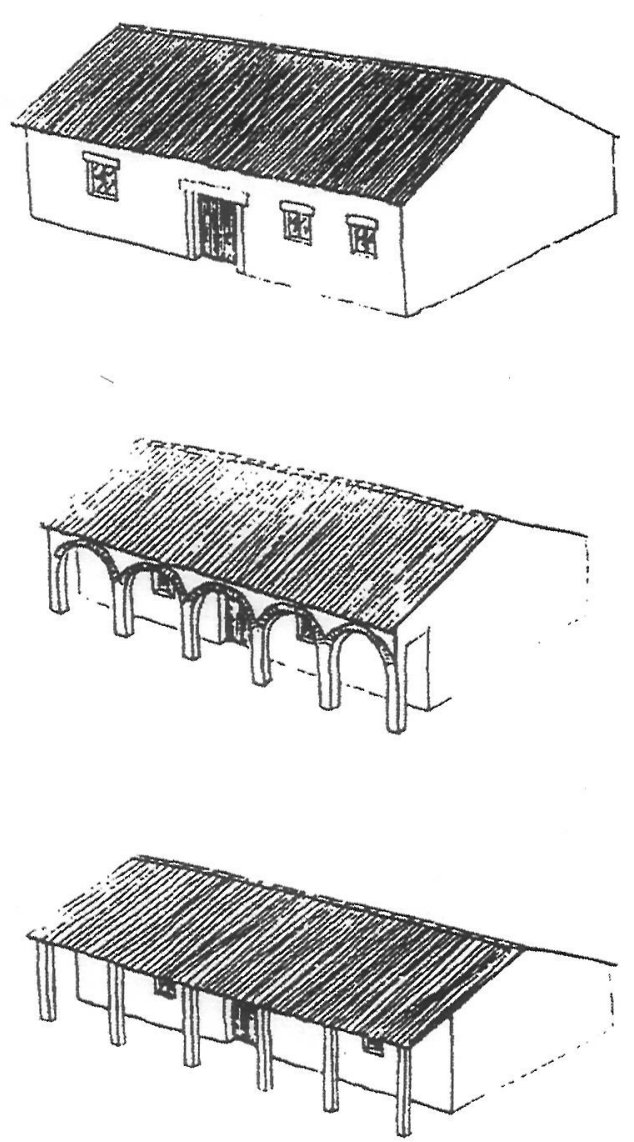

Figura 2: Fachadas de hospitales extremeños.
4- Moreno de Vargas, B (1992). Historia de la Ciudad de Mérida. Biblioteca Pública Municipal de Mérida. Séptima reedición. (Capítulo a libro).

5- García, M. (1978) "La piedad popular en Extremadura al final de la Edad Media". Hispania Sacra. (Artículo revista).

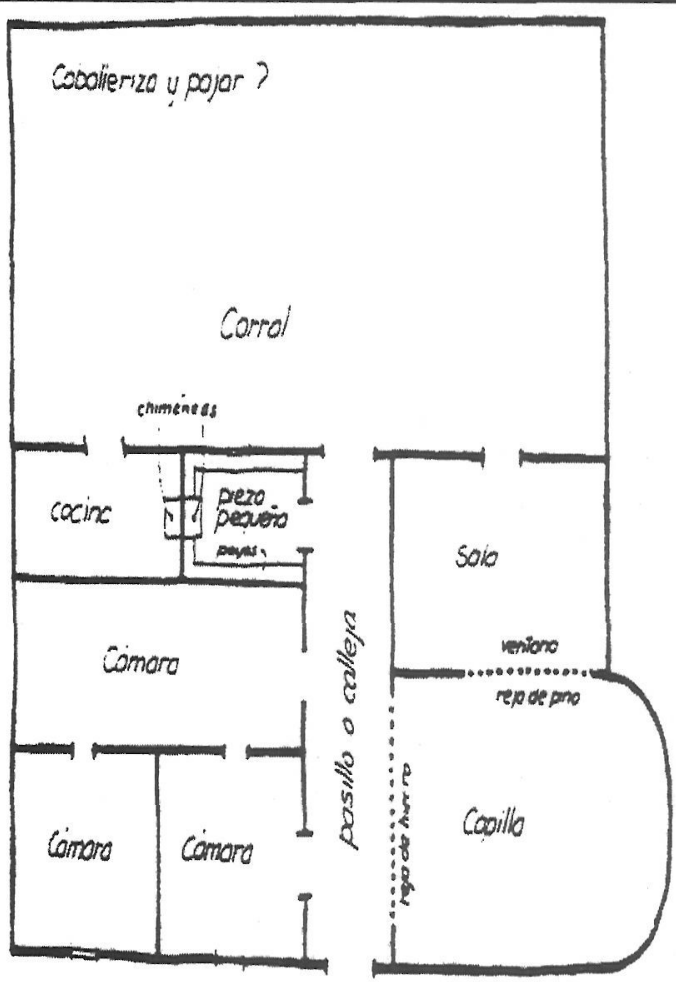

Figura 3: Hospital Nuestra Señora de Mérida 